\title{
Standard Ionization-Chamber Requirements for 250- to 500-Kilovolt X-Rays ${ }^{1}$
}

\author{
H. O. Wyckoff and F. S. Kirn ${ }^{2}$
}

\begin{abstract}
Information is provided on the correction factor required for the determination of the dose in roentgens of 250 - to 500-kilovolt X-rays from measurement with a free-air ionization chamber. Special emphasis is placed on the determination of each of the factors so that an over-all accuracy of 0.5 percent can be obtained.
\end{abstract}

\section{Introduction}

In 1953, the International Commission on Radiological Units recommended [1] the continued use of the roentgen up to $3 \mathrm{Mev}$ for the measurement of $\mathrm{X}$ - and gamma-ray dose. The physical requirements of standard ionization chambers for measuring roentgens in the X-ray region up to $250 \mathrm{kv}$ have already been reported $[2,3,4]$. The calibration of secondary instruments in at least two laboratories also seems to be in agreement [5] for cobalt-60 and radium, on the assumption of a fixed value for the emission constant (roentgens per milligram hour at $1 \mathrm{~cm}$ ) of radium. However, there is some question about the value of the emission constant for radium $[6,7]$ when its determination is based upon measurements with small cavity ionization chambers. In addition, many of the commercial secondary instruments show a difference in calibration of the order of 5 to 20 percent between $250-\mathrm{kv}$ X-rays and cobalt, so an interpolation of the calibration for the 300 - to $400-\mathrm{kv}$ X-ray therapy range seems risky. Extension of the range of standard instruments, at least up to cobalt energies, therefore seems desirable. The present report contains the design criteria for the construction of a standard chamber for 250- to $500-k v$ X-rays.

A plan view of one type of a standard free-air chamber is shown schematically in figure 1 . A parallel-plate system is contained in a radiationshielded box, B. A known area of the horizontal X-ray beam is defined by the diaphragm, D, so that a beam of photons passes centrally between the plates. A high potential (field strength of the order of $100 \mathrm{v} / \mathrm{cm}$ ) on plate $\mathrm{H}$, with respect to the other plates, sweeps out the ionization produced in the air between the plates. The ionization is measured for a length, $L$, of beam determined by the limiting lines of force to the edges of the collector, C. These lines are made straight and perpendicular to the collector by the guard plates, G, and auxiliary wires or strips, $W$. The latter are connected to a resistancedividing network to grade the potential uniformly across the end, top, and bottom gaps between $\mathrm{C}$ or $\mathrm{G}$, and H. Generally guard plates are not con-

1 Work supported by U. S. A tomic Energy Commission.

2 Now with Argonne National Laboratory, Idaho Falls, Idaho. sidered necessary above and below the collector because there the ionization density is low.

A measurement in roentgens requires, according to the definition [1], that one measure all of the ionization produced in air by the high-speed electrons that are themselves produced within the defined mass of air. Real difficulties arise in the measurement of the ionization as required by the definition, so the principle of electronic equilibrium is always used in practice.

Within a region under uniform irradiation, according to the principle of electronic equilibrium, the ionization produced outside of a specified mass, $m$, by high-speed electrons generated inside $m$ is compensated by ionization in $m$ produced by highspeed electrons generated outside of $m$. Thus electrons produced out to a distance from the mass equal to the electron range contribute to the ionization in the specified mass. Therefore in a standard chamber, the guard plates not only must be long enough to eliminate field distortion but also long enough to assure electronic equilibrium in the collecting region. For the latter, a distance equal to ralf the plate separation has been suggested [4] for X-rays up to $250 \mathrm{kv}$. However, for the higher-energy photons used here, the range of the electrons in the photon direction may be larger than the sideways range. A determination of the distance required for electronic equilibrium is therefore desirable.

Ionization is produced in the collecting region not only by the primary electrons, but also by electrons produced by secondary (scattered and fluorescent) photons. As this secondary ionization is not included in the roentgen definition, a correction is required to the measured ionization. However, this excess ionization tends to compensate for loss of primary ionization resulting from too small a plate separation and height, so that the required electrode separations are reduced by this effect.

In the calibration of a radiation instrument by a standard chamber, a substitution method is used. The exposure dose rate is determined from measurements of the ionization in the standard chamber, this chamber is removed, and the instrument being calibrated is placed at the position formerly occupied by the aperture of the standard chamber. Therefore, it is necessary to compute the exposure dose rate at the aperture from the ionization measurements. The factors required for such a conversion 


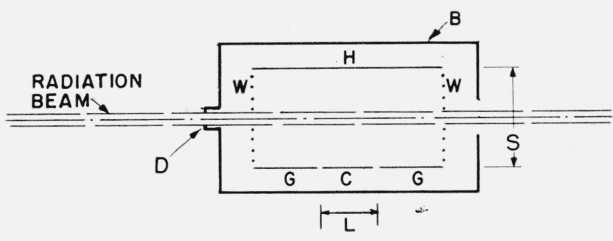

Figure 1. Schematic view of free-air ionization chamber showing a horizontal section through the radiation beam and chamber.

B, Shielded box; C, collector (length, L); D, diaphragm; G, guard plates; H, high-voltage plate; W, guard wires or strips; S, plate separation.

include a knowledge of the weighted position of origin of the high-speed electrons relative to the collecting volume and the air absorption between this position and the aperture.

Thus the information required for the proper design of a primary standard chamber is (1) the plate spacing, $S$ (fig. 1), required to assure dissipation of all of the energy of the secondary electrons in the air, (2) the criteria for producing a defined electric field so that this ionization is collected from a known length, $L$, of the beam, (3) the correction for the ionization produced by scattered photons, (4) the weighted position of origin of the high-speed electrons relative to the collecting region, and (5) the air absorption between this origin and the diaphragm. Data for item (2) have been obtained by Miller and Kennedy for the two-dimensional case [8]. Items (1) and (3) have been considered by Attix and DeLaVergne [4], by Kustner [2], and by Block [13], and item (1) by Kemp [3], for 50- to 250-kv X-rays. The present paper gives information on items (1), (3), (4), and (5) for 250- to 500-kv X-rays and moderate filtration. Work at higher energies is now under way at the Bureau.

\section{Apparatus}

A vertical, continuously pumped, end-grounded, 59-section X-ray tube was used. It was connected to the ground, center, and top of a 0 to $1,400-\mathrm{kv}$ generator [9]. The potential to the sections was graded uniformly by a series of resistors between the leads from the generator. The $90^{\circ}$ beam of X-rays (fig. 2) passed through the wall, W, (about 3-mm copper) of the X-ray tube, by a remotely controlled shutter, $\mathrm{S}$, through a collimator in front of the parallel-plate ionization chamber, and through a monitor ionization chamber, M. A focal-spot size of about $2-\mathrm{cm}$ diameter was obtained in the X-ray tube. Although this spot size was larger than desired, and sometimes shifted during the run, the monitor provided a means of normalizing the parallel-plate chamber readings because it measured most of the beam "seen" by the parallelplate chamber.

A lead-lined box contained the plates of the ionization chamber and limited the X-radiation measured by the chamber to that coming through the aperture. The box size was determined by pessimistic extrapolation from the requirements for lower energies. The lead protection required in the box was com- puted according to the method used in NBS Handbook $50[10]$ so that the ionization from the leakage radiation would be less than 0.1 percent of the ionization produced by the collimated beam. The front face of the chamber was shielded by 1-in.thick lead; the sides, top, and bottom by $3 / 8$-in.thick lead; and the back by 1/4-in.-thick lead. The aperture was cut in a 2 -in.-thick lead diaphragm. A 2-in.-thick lead plug over the aperture of the diaphragm (with the X-ray beam on) experimentally confirmed the adequacy of this protection. The snout on the box was provided to reduce the size of the box because it was anticipated that a scattering diaphragm would be necessary in order to stop radiation scattered from the primary diaphragm from entering the measuring volume. However, subsequent tests indicate, in agreement with tests of Attix and DeLaVergne [11] at lower energies, that this diaphragm is not needed.

The high-voltage plate was a single sheet of aluminum. The collector and field guards were fashioned from a single sheet $(90 \mathrm{~cm}$ by $90 \mathrm{~cm}$ ) of colloidalgraphite-coated polyethylene. A collector $(30 \mathrm{~cm}$ long by $80 \mathrm{~cm}$ high) was defined by lines scratched through the graphite with a sharp stylus. This collector was divided into seven 5-cm strips on each side of a central 10-cm strip by lines scratched through the graphite (see fig. 2). Any or all of them could be connected to the ionization-currentmeasuring instrument. When not so connected, they served as part of the guard system. There was a $5-\mathrm{cm}$ guard strip above and below the collector strip, and a 30-cm-long guard strip in front and in back of the collector strips. The plate separation could be varied from about 15 to $90 \mathrm{~cm}$, and the dimensions of the box permitted a movement of the plate system over a distance of about $1 \mathrm{~m}$ along the beam.

The distorting effect of the grounded box on the electric field was reduced by a system of guard strips and wires. As pointed out previously [8], the strips are preferable but wires are required where photon absorption is important. Double wires fastened to the inner and outer edges of the strips were therefore installed over an area of 50 by $50 \mathrm{~cm}$ at the front and back of the chamber system (see fig. 3). Strips (3 $\mathrm{mm}$ by $3 \frac{1}{2} \mathrm{~cm}$ ) were used over the remaining area and the top and bottom. With this system the grounded box produced less than 0.2 percent distortion ${ }^{3}$ when the plate system was located in the center of the box. To reduce the field distortion when the plate system was used near the front or back of the box, the double wires at both ends of the plate system were temporarily replaced by strips except for a central area of 15 by $50 \mathrm{~cm}$.

A 10-cm-i.d., 11ㅡ-mm thick, colloidal-graphitecoated, Bakelite tube was inserted between the plates to eliminate collection of the ionization produced by the primary photons. This tube was coaxial with the beam and extended in front of and behind the

${ }_{3}^{3}$ The distortion in the electric field caused by the proximity of the lead box was taken as one-half the fractional change in the ionization obtained for the box at ground potential and at the potential of the high-voltage plate. 


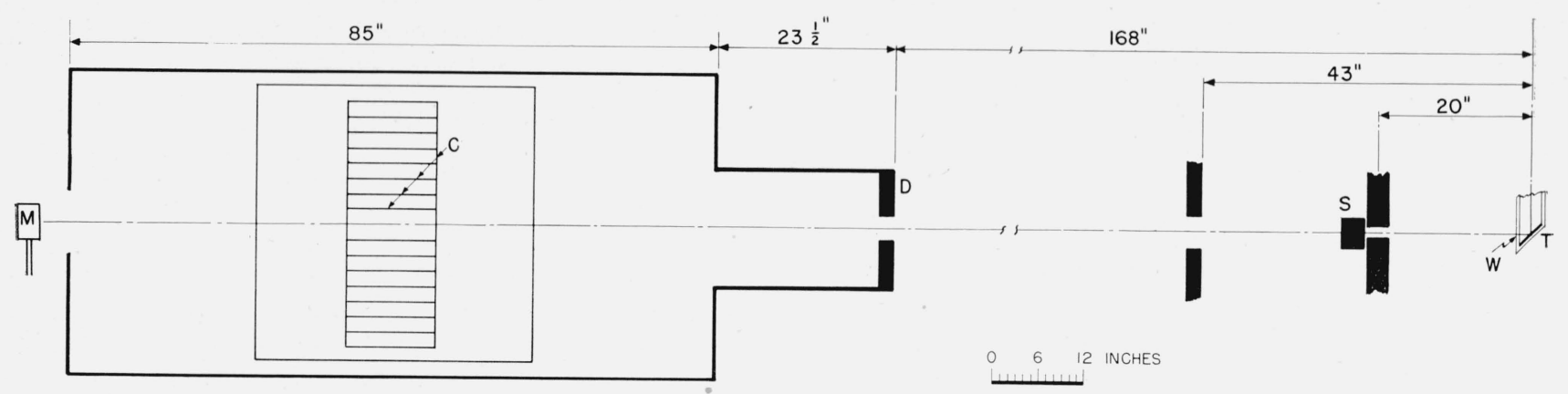

Figure 2. Elevation view of experimental arrangement.

T, X-ray target; S, shutter; D, diaphragm; C, collectors; M, monitor.

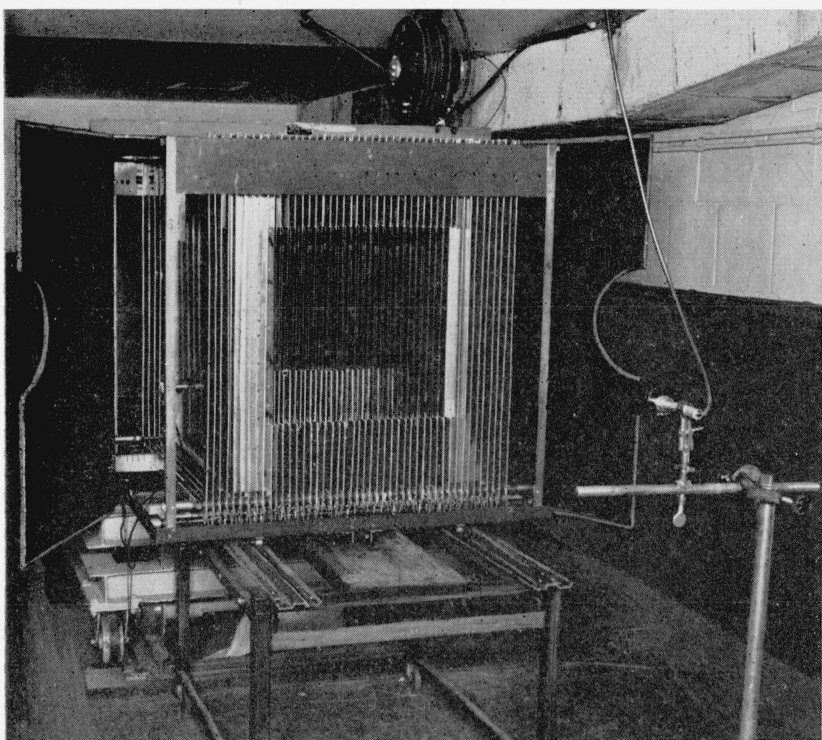

Figure 3. Part of free-air chamber showing plate system.

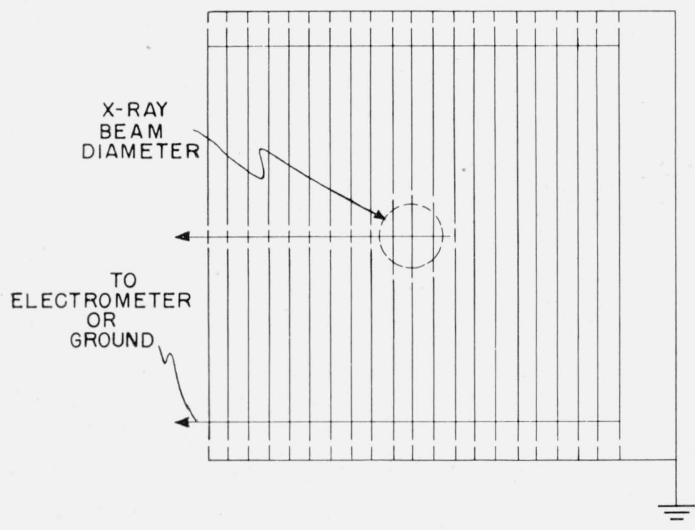

FIGURE 4. Schematic elevation of the center plane of the thread chamber.

Gaps in the straight lines indicate uncoated nylon threads. plate system. Scribed lines through the colloidal graphite along generatrices of the tube insulated the portions of the tube from each other and permitted them to be electrically connected to the guard wires. With this arrangement, the collected ionization is due only to scattered photons. The tube undoubtedly distorted the electric field in its vicinity but the total scattered photon contribution was small (less than 1 percent of the electron contribution from primary photons), so this distortion was assumed to be negligible.

A separate arrangement, similar to that used by Failla [12] to investigate the "electron cloud," was used to measure the growth of electronic equilibrium along the beam. A grid chamber of colloidalgraphite-coated nylon thread ( $\sim 0.2-\mathrm{mm}$ diameter) forming 5 parallel, 90 by $90-\mathrm{cm}$ planes was placed in the shielded box. The thread spacing was about $1 \mathrm{~cm}$ in each plane, and the planes were spaced about every $1.6 \mathrm{~cm}$ and perpendicular to the beam. The center plane (fig. 4) was graphite-coated to form two collectors insulated from each other and from ground by uncoated nylon thread. One of these collectors was slightly larger than the photon beam and concentric with it. The other included the rest of the plane. The planes immediately on each side of this center plane were connected to the collecting potential $(90 \mathrm{v})$. The outer two planes of thread were connected to ground and used to shield the collecting system from the grounded box.

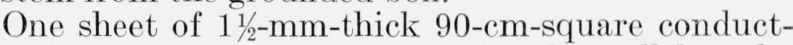
ing plastic was placed in front of and parallel to the plane of the grid chamber. A second was placed behind the chamber. Each sheet had a center hole slightly larger than the photon beam and each could be moved along the beam. As each of these sheets was thicker than the electron range, the ionization measured was produced to a first approximation ${ }^{4}$ by electrons from the air in the space between the sheets. The growth of ionization was explored by changing the location of the sheets.

\section{Procedure}

In order to eliminate the effects of X-ray output variation, simultaneous ionization measurements were made with the parallel-plate chamber and the

${ }_{4}^{4}$ The large size of the hole makes the exact position somewhat ambiguous. 


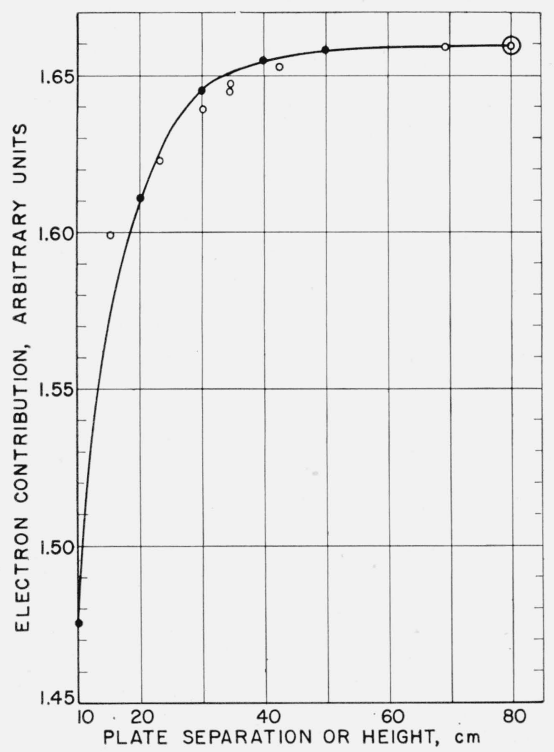

Figure 5. Ionization collected in air from 400-kv $X$-rays as a function of plate separation with a fixed height, and of height with a fixed plate separation.

$\mathrm{O}$, Height; $\bullet$ plate separation.

monitor. The charge from each was collected on a capacitor and the resulting potential measured under a null condition by a vibrating-reed electrometer. The ratio of the voltage on the parallel-plate capacitor to that on the monitor capacitor was proportional to the parallel-plate current for a fixed amount of monitor ionization. This averaging of the parallelplate current and the normalization by means of the monitor overcame the undesirable fluctuations in the X-ray output. Data were obtained at each $50 \mathrm{kv}$ in the 250 - to $500-\mathrm{kv}$ range.

The current collected by the collector plate is made up of two components, that part due to the ionization from high-speed electrons that are produced by the primary photons (primary contribution), and that part due to ionization from highspeed electrons produced by scattered and fluorescent photons (secondary contribution). The sum of the two contributions and the secondary contribution alone are measured $[2,4,13]$ so that the primary contribution can be determined by subtraction. In the present experiment, this sum was determined both by measuring the ionization collected by all of the collector strips $(80 \mathrm{~cm}$ high) for different plate separations, and by measuring the current collected by each strip for a fixed $80-\mathrm{cm}$ plate separation. The ionization collected by all of the strips at a given plate separation, $a$, should be equal to the ionization collected at $80-\mathrm{cm}$ plate separation by all of the strips up to a height equal to $a$. Figure 5 shows a representative comparison of the results by the two methods. Generally, the agreement was within 0.5 percent. However, the ionization density decreases rapidly with distance from the photon beam, so the individual strip method gives a more accurate measure of this density function. Therefore, the remainder of the data presented here were obtained by the strip method. Similar measurements were made with the Bakelite tube inserted to obtain the secondary contribution.

The current to each of the collectors of the grid chamber was measured separately. The one not used for collecting ionization was grounded. The variation of the ionization was determined as a function of the position of the conducting plastic sheets with respect to the grid chamber.

All measurements were obtained at room temperature and pressure, which averaged about $26^{\circ} \mathrm{C}$ and $760 \mathrm{~mm} . \mathrm{Hg}$.

\section{Results}

Figure 6 shows the primary contribution for 5-cmhigh layers of air at different vertical distances from the beam. The fractional loss in primary photon ionization (electron loss) at a particular height is obtained by summing the contributions from infinite plate height (obtained by linear extrapolation of the curves of fig. 6) to the plate height in question and dividing by the sum of all of the contributions out to infinity.

The diameter of the photon beam obviously affects the loss for a given plate separation. In figure 7 the percentage of electron loss is plotted against the plate separation minus the penumbra diameter $(6.3 \mathrm{~cm})$ at the center of the collecting region. On the $250-\mathrm{kv}$ curve are plotted data taken from results of Attix [11], ${ }^{5}$ where the penumbra was about 1.8 $\mathrm{cm}$ in diameter and the radiation filtration was approximately $1 \mathrm{~mm}$ of copper plus $4 \mathrm{~mm}$ of aluminum. This approximate method of treating the beam diameter seems to be adequate at least for these data, as the agreement of the electron contributions for the two experiments is generally within 0.1 percent. Furthermore, the difference is in the expected direction because the higher filtration used in the present experiment results in a smaller number of high-energy photoelectrons.

In order to compare the present data with those published earlier by Block [13] and to provide more easily usable data for other chamber configurations than the parallel-plate type, the electron loss and the scattered photon contribution were computed for cylindrical coordinates. Obviously the ionization collected from a ring of a given radius will be independent of angle, so only one variable is required. The contribution to each 5-cm ring was obtained from the results shown in figure 6 by the method previously outlined [4]. Figure 8 shows the electron loss per 5-cm-thick ring obtained at different inner radii. Here again the losses may be summed from infinity to a given radius to obtain the loss beyond that radius. The results are shown in figure 9 , where the beam size has been reduced to zero by subtracting the beam radius.

Figure 9 also shows some points computed from the data of Block [13] at $400 \mathrm{kv}$ and a filtration of

5 These plotted data are slightly different from those in table 2 of reference [4] because the plotted data are for an effectively infinite plate height. 


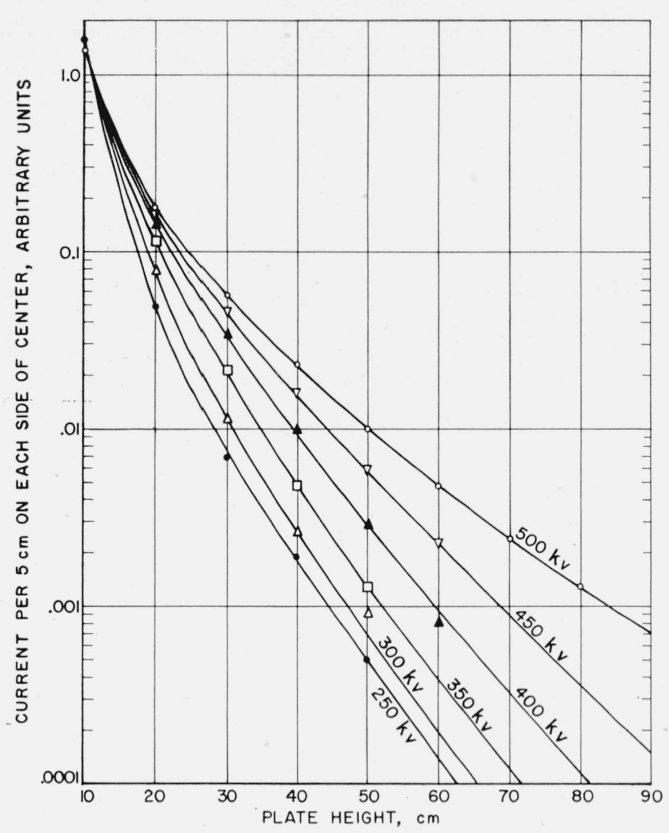

Figure 6. Primary electron-produced current per $5 \mathrm{~cm}$ of height of collector at different vertical distances from the beam and at various $X$-ray tube potentials.

The beam penumbra diameter was $6.3 \mathrm{~cm}$ in the middle of the collecting region.

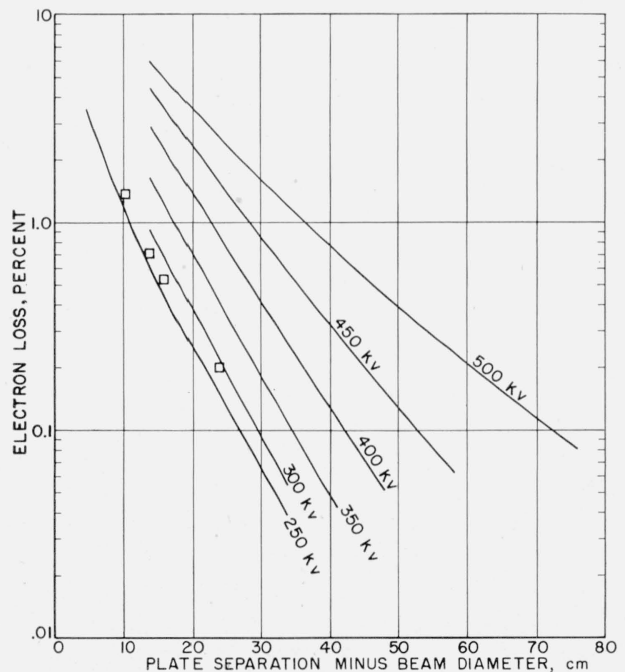

Figure 7. Percentage of primary electron ionization loss for different plate separations and a zero-diameter X-ray beam.

$\square$, Results of Attix for $250 \mathrm{kv}$.

$2.55 \mathrm{~mm}$ of tin plus $0.5 \mathrm{~mm}$ of copper plus $5.0 \mathrm{~mm}$ of aluminum. These points were obtained with a different filtration than that used for the present data; some of these had to be determined from smallscale drawings, the scattered photon contribution had to be computed from the present data, and the beam diameters were assumed to be the same for Block as for the present work. It is thus not surprising that these points differ by up to 0.5 percent from the present work.

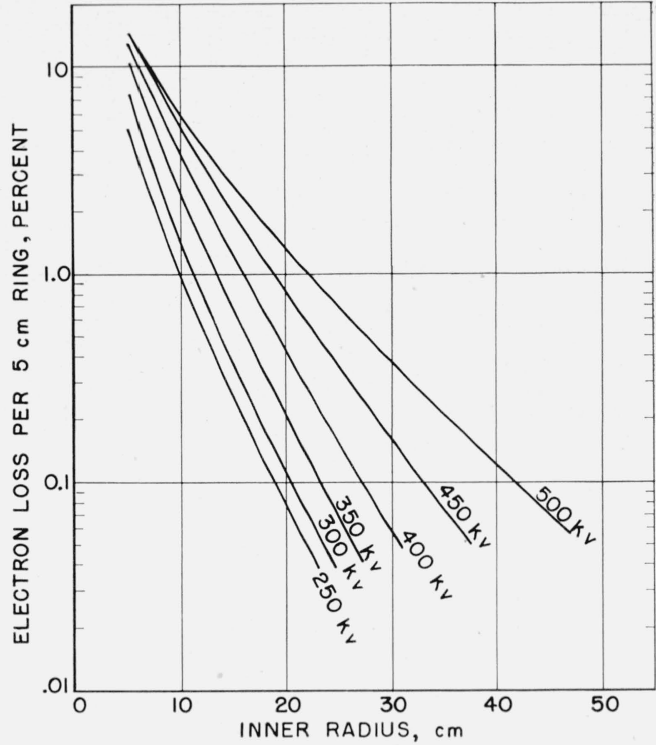

Figure 8. Percentage of primary electron ionization loss per 5-cm ring for different inner radii from the beam.

The beam penumbra diameter was $6.3 \mathrm{~cm}$ at the center of the collecting region.

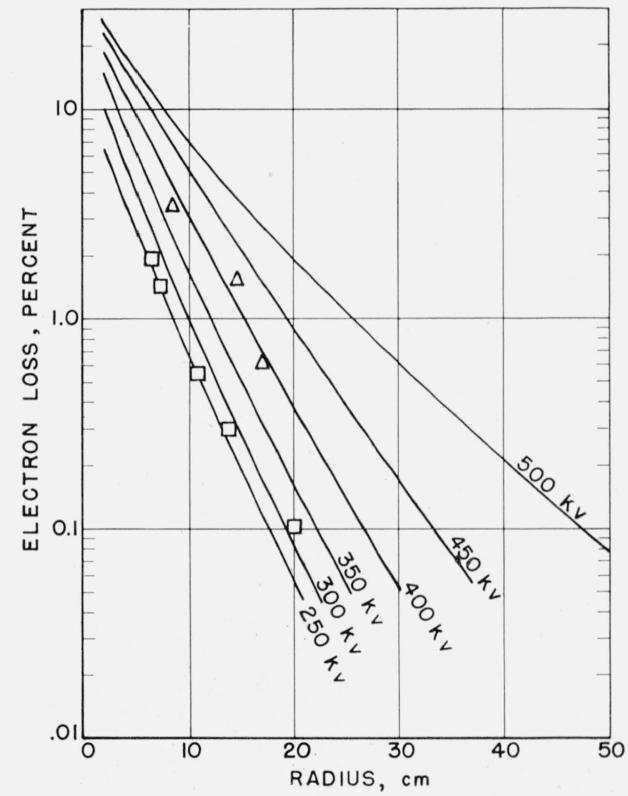

Figure 9. Percentage of primary electron ionization loss beyond different radii from the $X$-ray beam.

Results corrected to zero beam diameter. $\square$, Data of Attix [4] at $250 \mathrm{kv} ; \triangle$, data of Block [13] at $400 \mathrm{kv}$.

Figure 10 gives the radial distribution of the ionization from the secondary photons obtained from measurements made with the Bakelite tube in a fashion similar to that for the primary contribution. The contribution is given as a percentage of the total ionization from primary electrons. As the curves for various X-ray-tube potentials have a similar shape, only the $250-\mathrm{kv}$ curve is shown. The contribution at $300-\mathrm{kv}$ is obtained by multiplying the 


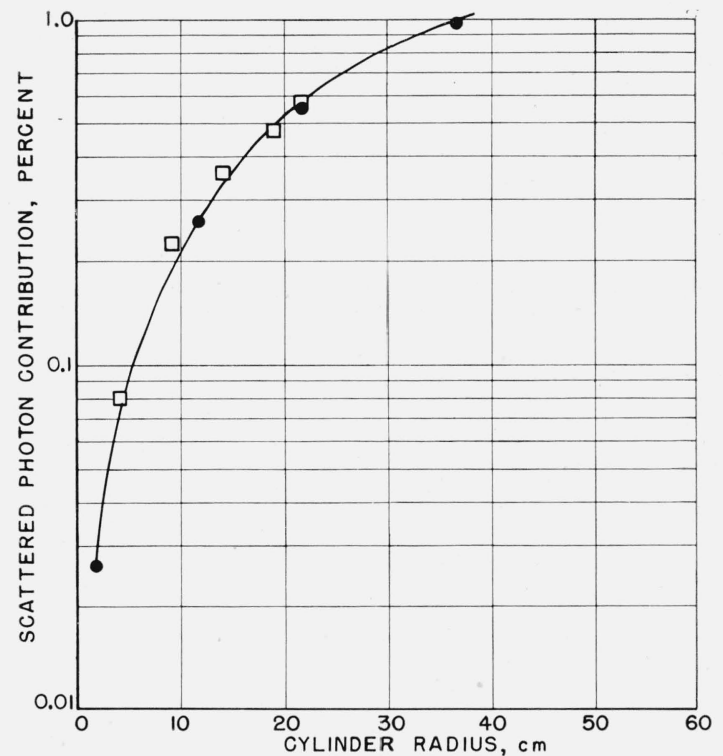

Figure 10. Contribution of secondary photon ionization in percentage of total primary electron ionization for different radii from a $250-k v$ zero-diameter $X$-ray beam.

, Present data; $\square$, data of Attix [11]. For $300 \mathrm{kv}$, multiply the ordinate by 0.9 ; for 400 and $500 \mathrm{kv}$, multiply by 0.8 .

ordinates of the curve by 0.9 ; at 400 and $500 \mathrm{kv}$, by 0.8 . It is interesting to note that the data obtained by Attix [11] at $250-\mathrm{kv}$ on a much smaller chamber give very nearly the same results. Here again the abscissa has been corrected for beam size by subtracting the beam radius.

The following table shows the air absorption per meter at various tube potentials obtained from ionization-chamber measurements with the chamber at the front and at the back of the box. Repeat measurements agreed to within \pm 0.2 percent. They also agreed to within 0.2 percent with the results of Block.

\begin{tabular}{|c|c|}
\hline $\begin{array}{c}\text { Tube } \\
\text { potential }\end{array}$ & $\begin{array}{c}\text { Air } \\
\text { absorption }\end{array}$ \\
\cline { 2 - 2 }$K v$ & $\% / m$ \\
250 & 1.7 \\
350 & 1.7 \\
500 & 1.3 \\
& \\
\hline
\end{tabular}

Figure 11 shows a typical "growth-of-electronicequilibrium" curve for the ionization collected by the outer electrode of the thread chamber with $500-\mathrm{kv}$ X-rays. The charge collected by the center electrode is 0.153 arbitrary unit, and is independent of the position of the plastic sheets. When the sheets are at a large distance from the collecting electrodes the total (equilibrium) ionization collected is $0.0444+0.032-0.153-0.0296$, or about 0.200 arbitrary unit. From these data for $500 \mathrm{kv}$, it is seen that $(0.153+0.029) / 0.200$, or about 90 percent,

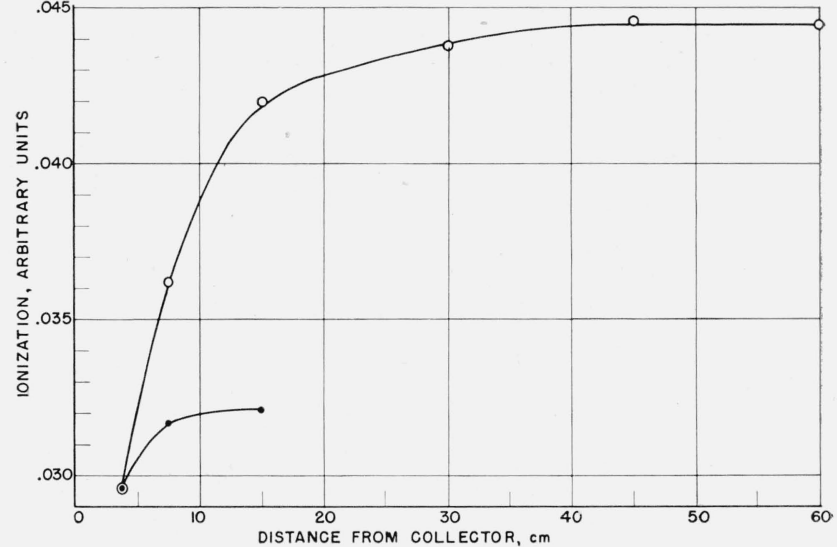

Figure 11. Results with the grid chamber with 500-kv X-rays.

The center electrode collected an ionization current of 0.153 arbitrary units. The abscissa gives the distance from one plastic sheet to the collector plane with the other sheet at $3.6 \mathrm{~cm}$ from the collector. Data for different distances of the front sheet are indicated by $\bigcirc$ and for the back sheet by

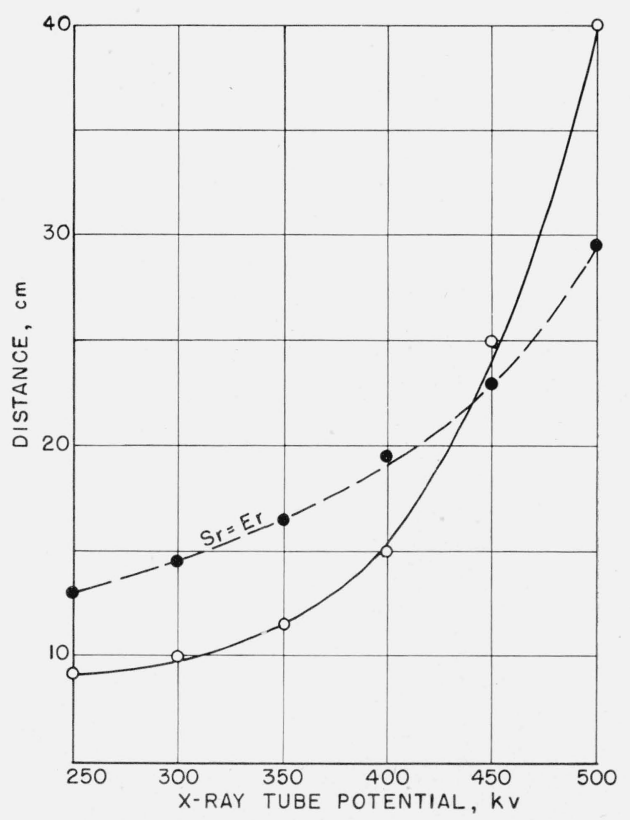

FIgURE 12. Approximate distance required for electronic equilibrium with medium filtration, and radii from photon beam at which the loss of primary electron ionization is compensated by gain of secondary radiation ionization.

0 , Distance; radii.

of this ionization results from electrons effectively originating within a few centimeters of the collecting region. Approximately 1 percent of the equilibrium ionization is produced by electrons originating up to about $15 \mathrm{~cm}$ behind, and about 90 percent by electrons originating up to $40 \mathrm{~cm}$ in front of the collecting region. The flux of gamma rays producing these high-speed electrons of course varies over the distance $(40+15 \mathrm{~cm})$ because of air absorption.

To a first approximation the absorption of X-rays in the air between the diaphragm and the origin of 
the high-speed electrons may be computed from the air-absorption coefficient and the distance between the diaphragm and the collecting region. A correction to this distance may be obtained from curves such as those in figure 11. For 500-kv X-rays the correction is

$$
\begin{aligned}
\frac{1}{0.200}\{-[+(0.0444) & \left.(40-3.7)-\int_{3.7}^{40} x_{f} d i\right] \\
+ & {\left.\left[(0.032)(15-3.7)-\int_{2.7}^{15} x_{b} d i\right]\right\}, }
\end{aligned}
$$

where 0.200 is the equilibrium ionization and $d i$ is an increment of ionization at a distance $x_{f}$ in front of the collector and $x_{b}$ behind the collector. On substituting values from the curve, this distance correction is found to be less than $1 \mathrm{~cm}$. As $8 \mathrm{~cm}$ of air attenuates the photon beam by only the order of 0.1 percent, the actual displacement is negligible. For all lower X-ray potentials the displacement is smaller. Therefore, the air-absorption correction in the 250- to 500-kv range should usually be computed from the center of the collector to the aperture.

Figure 12 shows the approximate distance required for electronic equilibrium. Also plotted are the distances equal to the radii from the beam, at which the loss of ionization from the primary electrons is just compensated by the gain of ionization from the secondary radiation. Thus, a collector-to-aperture distance, equal to the radius for compensation, is adequate for moderately filtered X-rays of $400 \mathrm{kv}$ and below but may be insufficient for 450 and $500 \mathrm{kv}$. It is seen from figure 11, for instance, that a decrease in the collector-to-aperture distance from 40 to 29.5 $\mathrm{cm}$ results in an error of about $(0.0444-0.0438) /$ 0.200 , or 0.3 percent.

\section{Summary}

The data required for the design of free-air standard chambers for measurement of 250 - to $500-\mathrm{kv} \mathrm{X}$ rays in roentgens have been obtained. The internal consistency of these data indicates that the errors of plate spacing can be estimated to about 0.2 percent. However, the much closer agreement between these results and those of Attix indicates that the uncertainty is probably less than 0.2 percent.

The assistance of George Dempsey, who helped set up the equipment and operated the X-ray unit, and of F. H. Attix, who participated in many of the technical discussions during the program of the experiment, is gratefully acknowledged.

\section{References}

[1] Recommendations of the International Commission on Radiological Units, Radiology $\mathbf{7 1 ,} 139$ (1954).

[2] H. Kustner, Ann. phys. 10, 616 (1931).

[3] L. A. W. Kemp and S. M. Hall, Brit. J. Radiol. 27, 219 (1954).

[4] F. H. Attix and L. DeLaVergne, J. Research NBS 53, 393 (1954) RP2558.

[5] H. O. Wyckoff, G. H. Aston, E. E. Smith, Brit. J. Radiol. 27, 325 (1954).

[6] G. N. Whyte, Nucleonics 1\%, No. 2, 18 (1954).

[7] L. V. Spencer and F. H. Attix, Radiology 64, 119A (1955).

[8] W. Miller and R. J. Kennedy, J. Research NBS 55, 291 (1955) RP2632.

[9] E. E. Charlton and H. S. Hubbard, Gen. Elec. Rev. 43, $272(1940)$.

[10] X-ray protection design, NBS Handb. 50 (1952).

[11] F. H. Attix, private communication.

[12] G. Failla and L. D. Marinelli, Am. J. Roentgenol. Radium Therapy 38, 312 (1937).

[13] W. I. Block, Ann. phys. 32, 701 (1938).

Washington, April 6, 1956. 